\title{
UM ESTUDO DOS FATORES CRÍTICOS DE SUCESSO EM PROJETOS DE TI GERENCIADOS POR ABORDAGENS ÁGEIS EM UMA INSTITUIÇÃO FINANCEIRA
}

\section{A STUDY OF CRITICAL SUCCESS FACTORS IN IT PROJECTS MANAGED BY AGILE APPROACHES IN A FINANCIAL INSTITUTION \\ UN ESTUDIO DE LOS FACTORES CRÍTICOS DE ÉXITO EN PROYECTOS DE TI GESTIONADOS POR ABORDAJES AGILES EN UNA INSTITUCIÓN FINANCIERA}

Pedro Jose Martins Alvarez Fernandes Mestre em Administração da UNINOVE pedromartins86@gmail.com

Roque Rabechini Jr

Pós-doutor e mestre em Administração pela Faculdade de Economia, Administração e Contabilidade da Universidade de São Paulo (FEA/USP). Professor do Programa de Pós-Graduação em Gestão de Projetos na Universidade Nove de Julho-UNINOVE rabechinijr@gmail.com https://orcid.org/0000-0002-6277-6571

\section{Editor Científico: José Edson Lara}

Organização Comitê Científico

Double Blind Review pelo SEER/OJS

Recebido em 07.06.2020

Aprovado em 08.10.2020

\footnotetext{
Este trabalho foi licenciado com uma Licença Creative Commons - Atribuição - Não Comercial 3.0 Brasil
} 


\section{Resumo}

Objetivo: Considerando a relação entre a abordagem ágil e o sucesso de projetos, o objetivo deste estudo foi avaliar os fatores críticos de sucesso dos projetos de tecnologia gerenciados por abordagem ágil em uma grande instituição financeira, analisando se os fatores identificados na literatura seriam suficientes para garantir o sucesso de tais projetos, assim como classificar os fatores críticos identificados em ordem de importância.

Metodologia: A metodologia utilizada foi um estudo de caso com abordagem exploratória.

Originalidade: Sabe-se que as técnicas e ferramentas pertencentes às abordagens de gerenciamento de projetos, exercem influência no resultado dos projetos. Com isso, visando aumentar ou estabilizar a taxa de sucesso desses, muitas empresas estão adotando a abordagem ágil, como alternativa à abordagem tradicional. Assim, considerando o sucesso de projetos gerenciados por abordagem ágil, focou-se na avaliação de projetos em uma empresa não projetizada e conservadora, características comumente encontradas em grandes instituições financeiras.

Principais resultados: Como resultado, foi possível verificar que os fatores identificados na revisão teórica são relevantes para o sucesso de projetos gerenciados por abordagem ágil, porém há a necessidade de agregar o controle das variáveis custo e escopo aos fatores identificados previamente. Ademais, identificou-se que os fatores envolvimento do cliente e estratégia de entrega, classificados na literatura como dois dos principais benefícios da abordagem ágil, foram classificados neste estudo como os fatores menos relevantes.

Contribuições teóricas: Este estudo possibilitou a criação de uma nova vertente para os fatores críticos de sucesso de projetos gerenciados por abordagem ágil e, pertencentes às instituições genuinamente conservadoras e inflexíveis.

Palavras-chave: Fatores críticos de sucesso, instituições financeiras, gerenciamento de projetos, ágil, projetos.

\section{Summary}

Objective: Considering the relationship between agile approach and success of projects, the aim of this study was to evaluate the critical success factors of IT projects managed by agile approach in a large financial institution, analyzing whether the factors identified in the literature would be sufficient to guarantee the success of such projects, as well as classify the critical factors identified in order of importance.

Methodology: The methodology used was a case study with an exploratory approach.

Originality: It is known that the techniques and tools belonging to the project management approaches, influence the results of the projects. Thus, in order to increase or stabilize the success rate of projects, many companies are adopting the agile approach, as an alternative to the traditional approach. Considering the success of projects managed by agile approach, this study focused on the evaluation of projects in a non-projected and conservative company, characteristics commonly found in large financial institutions. 
Main results: As a result, it was possible to verify that the factors identified in the theoretical review were relevant to the success of projects managed by agile approach, but it was necessary to add control of the cost and scope variables to the factors previously identified. In addition, it was identified that the factors customer involvement and delivery strategy, classified in the literature as two of the main benefits of the agile approach, were classified in this study as the least relevant factors.

Theoretical contributions: This study enabled the creation of a new approach to the critical success factors of projects managed by agile approach and, belonging to genuinely conservative and inflexible institutions.

Keywords: Critical success factors, financial institutions, project management, agile, projects.

\section{Resumen}

Objetivo: Considerando la relación entre la metodología ágil y el éxito de los proyectos, el objetivo de este estudio fue evaluar los factores críticos de éxito de proyectos de TI gestionados por metodología ágil en una gran institución financiera, analizando si los factores identificados en la literatura serían suficientes para garantizar el éxito de dichos proyectos, así como clasificar los factores críticos identificados en orden de importancia.

Metodología: La metodología utilizada fue un estudio de caso con enfoque exploratorio.

Originalidad: Se sabe que las técnicas y herramientas pertenecientes a las metodologías de gestión de proyectos, influyen en los resultados de los proyectos. Por lo tanto, para aumentar o estabilizar la tasa de éxito de los proyectos, muchas empresas están adoptando la metodología ágil como una alternativa a la metodología tradicional. Así, considerando el éxito de los proyectos gestionados por la metodología ágil, se centró en la evaluación de proyectos en una empresa conservadora y no proyectada, características habituales en las grandes instituciones financieras.

Principales resultados: Como resultado, fue posible verificar que los factores identificados en la revisión teórica son relevantes para el éxito de los proyectos gestionados por la metodología ágil, pero es necesario agregar el control de las variables de costo y alcance a los factores previamente identificados. Además, se identificó que los factores de participación del cliente y estrategia de entrega, clasificados en la literatura como dos de los principales beneficios de la metodología ágil, fueron clasificados en este estudio como los factores menos relevantes.

Aportes teóricos: Este estudio permitió la creación de un nuevo enfoque de los factores críticos de éxito de los proyectos gestionados por la metodología ágil y pertenecientes a instituciones genuinamente conservadoras e inflexibles.

Palabras clave: factores críticos de éxito, instituciones financieras, gestión de proyectos, ágil, proyectos. 


\section{INTRODUÇÃO}

Com a popularização dos projetos, tanto no setor público quanto no setor privado, diversos estudos destacam que a taxa de sucesso dos projetos ainda não é constante, considerando os altos índices de atrasos, correções, abandonos e rejeições de projetos, mesmo que já implementados (Chow \& Cao, 2008; Serrador \& Pinto, 2015).

Os projetos de tecnologia em uma instituição financeira são utilizados como instrumentos para implementar suas estratégias, portanto, o sucesso desses projetos é fundamental para garantir o avanço da organização a um novo patamar de serviços e produtos (Terlizzi et al., 2014). Entretanto, em 2015, somente 29\% dos projetos foram concluídos com sucesso, sendo que os projetos gerenciados por abordagens ágeis alcançaram taxas de sucesso melhores (39\%), quando comparados aos projetos gerenciados por meio de abordagens tradicionais (11\%) (The Standish Group, 2015).

Com o intuito de maximizar o impacto positivo dos projetos, criou-se as abordagens de gerenciamento de projetos, inicialmente as denominadas tradicionais, com processos mais rígidos e lineares e, posteriormente, as abordagens ágeis, essas que são mais flexíveis e adaptativas (Boehm, 2000, 2002).

Serrador e Pinto (2015) comentam que as abordagens ágeis surgiram como alternativa para $\mathrm{o}$ atendimento das necessidades citadas anteriormente, assim como contrastam com as abordagens tradicionais, visto que enfatizam o desenho contínuo da solução, escopo flexível e interação constante dos stakeholders. Destacam ainda que um processo de desenvolvimento rígido, tal como é utilizado pelas abordagens tradicionais, pode impactar diretamente o sucesso dos projetos, por meio de retrabalhos, inflexibilidade e insatisfação dos stakeholders. Senapathi e Drury-Grogan (2017) enfatizam a ascensão das abordagens ágeis no gerenciamento de projetos relacionados ou não à tecnologia, sendo consideradas viáveis e tornando-se um dos principais tópicos pesquisados do gerenciamento de projetos.

Terlizzi at al. (2014) enfatizam que a competitividade no setor financeiro, motivada pelo surgimento das Fintechs, faz com que as grandes organizações desse setor necessitem de tempestividade na tomada de decisão, assim como uma maior flexibilidade na definição e implementação de suas estratégias.

Dessa forma, por meio de um estudo de caso com abordagem exploratória, este artigo pretende analisar os principais fatores que influenciaram o sucesso dos projetos de 
tecnologia gerenciados por abordagem ágil em uma grande instituição financeira brasileira, evidenciando se os fatores identificados na literatura foram suficientes para garantir o sucesso desses projetos.

\section{Referencial Teórico}

\subsection{Gerenciamento de projetos}

Segundo Turner (2008), 30\% da economia global é baseada em projeto. O autor ainda destaca a crescente quantidade de estudos envolvendo o gerenciamento de projetos, gerando mudanças relevantes nas práticas desta área de conhecimento e, consequentemente, gerando alternativas para um gerenciamento eficiente dos projetos. Patah e Carvalho (2012) comentam a existência de diversos modelos de referência em gerenciamento de projetos, esses que auxiliam os profissionais e as organizações nas atividades de gerenciamento, assim como contribuem para uma melhor taxa de sucesso dos projetos. Os métodos mais difundidos são disponibilizados por associações e institutos dedicados ao estudo de projetos, como descrito na

\section{Tabela 1.}

\section{Tabela 1}

Principais associações de gerenciamento de projetos e seus métodos

\begin{tabular}{llll}
\hline Instituto & Guia de Conhecimento & País Foco & Objetivo \\
\hline $\begin{array}{l}\text { Institute (PMI) } \\
2 \text { - Office of Government }\end{array}$ & $\begin{array}{l}\text { Project Management Body of } \\
\text { Knowledge (PMBoK) }\end{array}$ & EUA & $\begin{array}{l}\text { Gestão geral de } \\
\text { projetos }\end{array}$ \\
Eommerce (OGC) & Environments (PRINCE2) & Reino Unido & $\begin{array}{l}\text { Gestão de projetos de } \\
\text { sistemas de informação }\end{array}$ \\
$\begin{array}{l}\text { Management Association } \\
\text { (IPMA) }\end{array}$ & $\begin{array}{l}\text { ICB - IPMA Competence } \\
\text { Baseline }\end{array}$ & $\begin{array}{l}\text { União } \\
\text { Europeia }\end{array}$ & $\begin{array}{l}\text { Gestão geral de } \\
\text { projetos }\end{array}$ \\
$\begin{array}{l}\text { P - Australian Institute of } \\
\text { Project Management (AIPM) }\end{array}$ & $\begin{array}{l}\text { AIPM - Professional } \\
\text { Competency Standards for }\end{array}$ & Austrália & $\begin{array}{l}\text { Gestão geral de } \\
\text { projetos }\end{array}$ \\
$\begin{array}{l}\text { Project Management } \text { - Association for Project } \\
\text { Management (APM) }\end{array}$ & APM Body of Knowledge & Reino Unido & $\begin{array}{l}\text { Gestão geral de } \\
\text { projetos }\end{array}$ \\
$\begin{array}{l}\text { F - Japan Project Management } \\
\text { Forum (JPMF) }\end{array}$ & $\begin{array}{l}\text { ENAA Model Form- } \\
\text { International Contract for } \\
\text { Process Plant Construction }\end{array}$ & Japão & $\begin{array}{l}\text { Gestão de projetos de } \\
\text { construções }\end{array}$ \\
\hline
\end{tabular}

Fonte: Adaptado de Patah e Carvalho (2012).

As abordagens tradicionais de gerenciamento, inicialmente utilizadas na maior parte dos projetos, tornaram-se ineficientes para alguns tipos de projetos, considerando sua 
linearidade e inflexibilidade. Assim, como alternativa para as fragilidades identificadas, surgiram as abordagens ágeis de gerenciamento de projetos, focando em ciclos interativos e entregas constantes (Boehm \& Turner, 2003; Highsmith \& Cockburn, 2001).

\subsection{Abordagens ágeis de gerenciamento de projetos}

Conforme mencionado por Anderson (2003), ágil significa que algo é flexível e tempestivo, assim, abordagem ágil pode ser considerada como a habilidade para sobreviver em uma atmosfera de constante mudança. Segundo Serrador e Pinto (2015), a abordagem ágil é predominantemente um fenômeno de tecnologia, porém seu sucesso fez com que essa abordagem fosse expandida para projetos não relacionados a tecnologia.

A abordagem ágil abrange um conjunto técnicas, modelos e métodos com o objetivo de conviver com o risco inerente a ambientes dinâmicos, por meio da flexibilidade, atividades interativas e entregas constantes, tanto para atividades relacionadas ao gerenciamento de projetos quanto para atividades de desenvolvimento de software (Boehm \& Turner, 2003; Highsmith \& Cockburn, 2001). Assim, tal abordagem consolidou-se como uma solução para os problemas identificados pelas abordagens tradicionais, como o excesso de burocracia e inflexibilidade das mudanças de escopo, por meio da eliminação de barreiras e interação dos stakeholders (Boehm \& Turner, 2003).

Destaca-se que as abordagens ágeis apenas ganharam popularidade após a elaboração do 'Manifesto Ágil', este que foi descrito por gestores de projetos experientes em meados de 2001, propondo práticas para o gerenciamento ágil de projetos e focando nos valores descritos na Tabela 2.

\section{Tabela 2}

\begin{tabular}{ll} 
Valores do Manifesto Ágil \\
\hline Identificação & Descrição \\
\hline 1 & Indivíduos e interações mais que processos e ferramentas. \\
2 & Software em funcionamento mais que documentação abrangente. \\
3 & Colaboração com o cliente mais que negociação de contratos. \\
4 & Responder a mudanças mais que seguir um plano. \\
\hline
\end{tabular}

Fonte: Adaptado de Dybå e Dingsøyr (2008).

A partir desses valores, sugiram os princípios do manifesto ágil, conforme apresentado na Tabela 3, que juntamente com os valores mencionados anteriormente formam os alicerces da filosofia ágil (Dybå \& Dingsøyr, 2008): 


\section{Tabela 3}

\begin{tabular}{|c|c|}
\hline Identificação & Descrição \\
\hline 1 & $\begin{array}{l}\text { Nossa maior prioridade é satisfazer o cliente, através da entrega adiantada e contínua de } \\
\text { software de valor. }\end{array}$ \\
\hline 2 & $\begin{array}{l}\text { Aceitar mudanças de requisitos, mesmo no fim do desenvolvimento. Processos ágeis se } \\
\text { adequam a mudanças, para que o cliente possa tirar vantagens competitivas. }\end{array}$ \\
\hline 3 & $\begin{array}{l}\text { Entregar software funcionando com frequência, na escala de semanas até meses, com } \\
\text { preferência aos períodos mais curtos. }\end{array}$ \\
\hline 4 & $\begin{array}{l}\text { Pessoas relacionadas à negócios e desenvolvedores devem trabalhar em conjunto e } \\
\text { diariamente, durante todo o curso do projeto. }\end{array}$ \\
\hline 5 & $\begin{array}{l}\text { Construir projetos ao redor de indivíduos motivados. Dando a eles o ambiente e suporte } \\
\text { necessário, e confiar que farão seu trabalho. }\end{array}$ \\
\hline 6 & $\begin{array}{l}\text { O método mais eficiente e eficaz de transmitir informações para, e por dentro de um time de } \\
\text { desenvolvimento, é através de uma conversa cara a cara. }\end{array}$ \\
\hline 7 & Software funcional é a medida primária de progresso. \\
\hline 8 & $\begin{array}{l}\text { Processos ágeis promovem um ambiente sustentável. Os patrocinadores, desenvolvedores e } \\
\text { usuários, devem ser capazes de manter indefinidamente, passos constantes. }\end{array}$ \\
\hline 9 & Contínua atenção à excelência técnica e bom design, aumenta a agilidade. \\
\hline 10 & Simplicidade: a arte de maximizar a quantidade de trabalho que não precisou ser feito. \\
\hline 11 & As melhores arquiteturas, requisitos e designs emergem de times auto organizáveis. \\
\hline 12 & $\begin{array}{l}\text { Em intervalos regulares, o time reflete em como ficar mais efetivo, então, se ajustam e } \\
\text { otimizam seu comportamento de acordo. }\end{array}$ \\
\hline
\end{tabular}

Fonte: Adaptado de Dybå e Dingsøyr (2008).

Dybå \& Dingsøyr (2008) destacam a existência de diversos estudos comparando as características e eficácia das abordagens tradicionais e ágeis. Portanto, a Tabela Erro! Fonte de referência não encontrada. sumariza as principais diferenças entre essas abordagens, revelando que a abordagem tradicional se concentra nos processos e requisições detalhadas previamente, enquanto as abordagens ágeis fomentam o dinamismo e a comunicação das pessoas.

\section{Tabela 4}

Principais características das abordagens tradicionais e ágeis

\begin{tabular}{|c|c|c|}
\hline Categorias & Abordagem Tradicional & Abordagem Ágil \\
\hline 1 - Suposições fundamentais & $\begin{array}{l}\text { Sistemas totalmente especificados, } \\
\text { previsíveis e desenvolvido por meio } \\
\text { de um planejamento detalhado. }\end{array}$ & $\begin{array}{l}\text { Desenvolvido por times pequenos, } \\
\text { utiliza os princípios de desenho } \\
\text { contínuo da solução e baseado nos } \\
\text { feedbacks e mudanças. }\end{array}$ \\
\hline 2 - Estilo de gerenciamento & Comando e controle. & Liderança e colaboração. \\
\hline 3 - Gestão do conhecimento & Explícito. & Tácito. \\
\hline 4 - Comunicação & Formal. & Informal. \\
\hline 5 - Modelo de desenvolvimento & $\begin{array}{l}\text { Modelo de ciclo de vida (ex: } \\
\text { cascata). }\end{array}$ & Modelo de entrega evolucionária. \\
\hline 6 - Estrutura organizacional & $\begin{array}{l}\text { Mecanicista (burocrático e com alta } \\
\text { formalização). Direcionado para }\end{array}$ & $\begin{array}{l}\text { Orgânica (flexível, participativa e } \\
\text { colaborativa). Direcionada para }\end{array}$ \\
\hline
\end{tabular}




\begin{tabular}{lll}
\hline Categorias & Abordagem Tradicional & Abordagem Ágil \\
\hline & grandes empresas. & pequenas e médias empresas. \\
7 - Controle de qualidade & $\begin{array}{l}\text { Planejamento detalhado e controle } \\
\text { minucioso. }\end{array}$ & $\begin{array}{l}\text { Controle contínuo dos } \\
\text { requerimentos, desenho e solução. }\end{array}$ \\
\hline
\end{tabular}

Fonte: Adaptado de Dybå e Dingsøyr (2008).

\subsection{Fatores críticos de sucesso (fes)}

O monitoramento tradicional do sucesso do projeto, geralmente é realizado com base em sua eficiência, considerando as variáveis tempo, custo e escopo, também conhecidas como restrição tripla (Serrador \& Pinto, 2015). Destaca-se que diversos autores defendem a existência de uma forte relação entre a eficiência do projeto e seu sucesso, afirmando que, apesar de não garantir o sucesso do projeto, as variáveis contempladas em sua eficiência não podem ser negligenciadas (Kloppenborg et al., 2009; Serrador \& Turner, 2014; Shenhar et al., 1997). O insucesso dos projetos e os problemas identificados na seleção e aplicação dos métodos utilizados no gerenciamento de projetos são discutidos por especialistas e pesquisadores desde a década de 1980 (Boehm, 1984, 1988; Heninger, 1980).

Corroborando com as informações descritas anteriormente, The Standish Group (2015), por meio do relatório Chaos Report, informa que muitos projetos atenderam a restrição tripla e não foram classificados como projetos de sucesso, visto que não agregaram valor às organizações ou não geraram a satisfação dos stakeholders. Ademais, um projeto conduzido de forma efetiva não necessariamente gera resultados considerados de sucesso, ou seja, um gerenciamento de sucesso não garante $\mathrm{o}$ atendimento dos objetivos do projeto $\mathrm{e}$ consequentemente a satisfação das partes interessadas (Wit, 1988).

Os estudos sobre o sucesso dos projetos resultaram na identificação dos fatores críticos de sucesso (FCS), esses que são definidos como áreas, cujos resultados satisfatórios são imprescindíveis para o sucesso do projeto e consequentemente da organização (Rockart, 1979). Com isso, identifica-se a necessidade de mapeamento dos fatores críticos de sucesso em projetos gerenciados por abordagens ágeis. Assim, considerando a literatura relacionada a fatores críticos de sucesso e abordagens ágeis, Chow e Cao (2008) identificaram diversos fatores, classificando-os em cinco dimensões, a saber: organizacional, pessoas, processo, técnico e projeto, conforme descrito na Tabela 4. 


\section{Tabela 4}

Fatores críticos de sucesso (FCS)

\begin{tabular}{ll}
\hline Dimensão & Fator \\
\hline Organizacional & 1. Comprometimento de gestão \\
& 2. Ambiente organizacional \\
Pessoas & $\begin{array}{l}\text { 3. Ambiente da equipe } \\
\text { 4. Capacidade da equipe }\end{array}$ \\
5rocesso & 6. Envolvimento do cliente \\
& 7. Processo de gerenciamento de definição de projetos \\
Técnico & 8. Técnicas ágeis \\
& 9. Estratégia de entrega \\
Projeto & 10. Natureza do projeto \\
& 11. Tipo do projeto \\
12. Cronograma do projeto
\end{tabular}

Fonte: Adaptado de Chow e Cao (2008).

Para a dimensão "organizacional”, os autores consideraram itens relacionados ao ambiente corporativo, tais como: cultura, estrutura, sponsorship e reconhecimentos. Por outro lado, a dimensão "pessoas” está voltada para a equipe, abordando aspectos de motivação, capacidade, relacionamento e engajamento. A dimensão "processo" trata dos processos definidos na organização, no que tange a comunicação, envolvimento dos stakeholders e a orientação dos processos às abordagens ágeis. Com foco no conhecimento dos envolvidos no projeto e nos procedimentos técnicos, a dimensão "técnico" aborda aspectos relacionados a capacitação, documentação e atividades técnicas. Por fim, a dimensão "projeto" considera as características do projeto, tais como natureza, tipo, riscos, entre outras.

\section{METODOLOGIA}

Este estudo trata-se de um relato técnico, cujo objetivo é descrever uma experiência real em contexto organizacional. Apesar de seu teor prático, seguiu-se todo o rigor científico e metodológico sugerido por Biancolino, Kniess, Maccari e Rabechini Jr (2012).

Considerando como unidade de análise os projetos de tecnologia de um dos maiores bancos da américa latina, este estudo possui uma abordagem exploratória, utilizando a pesquisa bibliográfica como estratégia de pesquisa. Além disso, os dados foram coletados 
por meio de análise de dados secundários dos projetos e entrevistas não estruturadas com os gestores responsáveis pelos projetos selecionados.

A pesquisa exploratória tem como principal objetivo o entendimento aprofundado do conhecimento sobre o problema a fim de torná-lo evidente (Gil, 2010). Por outro lado, a pesquisa bibliográfica geralmente utiliza fontes de dados secundários, sendo fundamental para pesquisas científicas de qualquer natureza, visto que o objetivo contempla o entendimento aprofundado de um assunto ou problema a partir de um referencial teórico. Ademais, entrevistas não estruturadas são definidas como uma técnica de pesquisa, cujo principal objetivo é coletar informações, dados e evidencias, que auxiliem no entendimento e compreensão das questões e situações, baseando-se na percepção dos entrevistados (Martins \& Theóphilo, 2009).

Destaca-se que os resultados obtidos com a pesquisa bibliográfica, possibilitaram a identificação da viabilidade e relevância do tema abordado, seleção do método mais apropriado, assim como serviram de insumo para a elaboração das questões aplicadas nas entrevistas.

Considerando a quantidade e diversidade de projetos existentes no portfólio da instituição avaliada, aplicou-se alguns filtros ne seleção dos projetos utilizados nesta pesquisa, permitindo uma maior assertividade e confiabilidade dos resultados, a saber: (i) projetos concluídos até a data de aplicação da pesquisa; (ii) projetos inteiramente gerenciados por abordagens ágeis; e (iii) projetos cujos gestores ainda fazem parte do quadro de colaboradores da instituição.

Por fim, destaca-se que foi utilizado o seguinte roteiro para a elaboração deste trabalho: (i) elaboração da questão de pesquisa com base na literatura relacionada e em situações vivenciadas pelo autor na organização avaliada; (ii) seleção dos projetos a serem avaliados; (iii) coleta dos dados secundários gerados pelos projetos concluídos; (iv) entrevistas não estruturadas com os gestores responsáveis pelos projetos selecionados anteriormente; (v) análise dos dados obtidos; e (vi) conclusão da pesquisa.

\section{APRESENTAÇÃO E DISCUSSÃO DOS RESULTADOS}

O estudo de caso foi realizado com base nas informações de cinco projetos de tecnologia de uma grande instituição financeira brasileira, cujo objetivo era melhorar a experiência dos usuários por meio de mudanças nos canais digitais, tais como internet 
banking e mobile banking. Ressalta-se que a instituição avaliada não autorizou a publicação da quantidade de projetos concluídos, assim como os detalhes dos projetos selecionados e o próprio nome da empresa. Com isso, respeitando o sigilo das informações, os autores referenciaram a empresa e os projetos de uma maneira mais genérica.

Atualmente, a instituição avaliada possui uma área destinada exclusivamente à manutenção dos canais digitais, estes que são considerados diferenciais estratégicos no setor financeiro, visto que com exceção das Fintechs, a maioria das empresas pertencentes ao setor mencionado, são empresas mais conservadoras e, consequentemente, inflexíveis e burocráticas. O termo Fintech surgiu da união das palavras em inglês financial e technology. Além disso, as fintechs são majoritariamente empresas emergentes, também conhecidas como startups, que trabalham a inovação e otimização de serviços do sistema financeiro (Paternoster et al., 2014; Terlizzi et al., 2014).

Capturou-se durante as entrevistas que, anteriormente, todos os projetos de tecnologia eram gerenciados por meio das abordagens tradicionais de gerenciamento de projetos, focando exclusivamente nas técnicas descritas no PMBoK, criado pelo Project Management Institute, conhecido pelo acrônimo PMI. Entretanto, os projetos selecionados para este estudo, assim como seus semelhantes, foram gerenciados por meio de abordagens ágeis. Segundo os gestores entrevistados, a transição das abordagens tradicionais para as abordagens ágeis foi necessária, pois os projetos relacionados aos canais digitais impactam diretamente o cliente, requerendo um retorno mais tempestivo, esse que se torna mais factível por meio das abordagens ágeis de gerenciamento de projetos. Enfatizam ainda que, em linhas gerais, os projetos utilizam uma mescla de abordagens já disseminadas no mundo corporativo, tais como Scrum e Kanban. Por fim, comentam que os projetos mais longos, tais como os projetos de infraestrutura, ainda são gerenciados por abordagens tradicionais ou híbridas, visto que estes projetos em sua grande maioria não focam nos canais digitais, ou seja, não impactam diretamente os clientes e não necessitam de um retorno imediato.

Destaca-se que para a realização dos projetos selecionados para este estudo, criouse equipes multidisciplinares, também chamadas internamente de comunidades, contendo os papéis destacados na Tabela 5. Com exceção dos colaboradores que exercem o papel de Product Owner, todos os demais colaboradores, a saber Tech Lead, Team Lead e Team Member, estão alocados na estrutura de tecnologia, visto que a grande maioria das demandas são melhorias no ambiente tecnológico. Assim, considerou-se na coleta de dados, os colaboradores com algum viés de gestão e pertencentes à área de tecnologia da empresa. Em 
outras palavras, selecionou-se os colaboradores que exerceram os papéis de Tech Lead e Team Lead nos projetos considerados na amostra deste estudo, totalizando dez entrevistados. Enfatiza-se que a seleção de papéis pertencentes à área de tecnologia, corrobora com o objetivo deste estudo que é analisar os fatores críticos de sucesso sob a perspectiva de projetos de tecnologia.

Cada projeto possui ainda um gerente de projetos associado, porém, optou-se por não selecionar os colaboradores que exerceram esta função, devido à limitação de detalhes que este papel possui com relação ao dia-a-dia dos projetos.

\section{Tabela 5}

Papéis contidos nas equipes multidisciplinares

\begin{tabular}{ll}
\hline Papéis & Cargos \\
\hline Product Owner & 1. Analista de produtos/negócio \\
Tech Lead & 2. Engenheiro de sistema \\
& 3. Arquiteto de soluções \\
Team Lead & 4. Gestor de projeto \\
Team member & 5. Desenvolvedor \\
& 6. Analista de qualidade \\
& 7. Engenheiro de testes \\
& 8. Engenheiro de suporte \\
& 9. Analista de user experience (UX) \\
10. Arquiteto de dados
\end{tabular}

Fonte: Elaborado pelos autores.

Além das informações capturadas nas entrevistas com os colaboradores participantes dos projetos selecionados, considerou-se as informações contidas em bases secundárias relacionadas aos projetos, tais como indicadores de custo e prazo, dashboards e demais informações inseridas no software corporativo utilizado para realizar o gerenciamento dos projetos de tecnologia. Todas essas informações são obrigatoriamente armazenadas e constantemente avaliadas, por auditorias internas e externas, assim como são discutidas em comitês executivos internos, demonstrando a integridade de todos esses dados.

Os entrevistados classificaram os cinco projetos avaliados como casos de sucesso, visto que atenderam as expectativas dos stakeholders, assim como foi possível capturar os benefícios previstos, fatos que foram corroborados pelos dados capturados no software corporativo de gerenciamento de projetos. Destacaram que, por se tratar de projetos de tecnologia gerenciados por abordagens ágeis, as calculadoras de investimentos, contendo os racionais dos benefícios e investimentos, foram elaboradas de forma contínua, ou seja, durante a própria execução dos projetos. Para a captura do retorno previsto, os gestores consideram o feedback da área de negócio, considerada como um cliente interno, pois a 
maioria dos projetos são oriundos de solicitações de negócios. Além disso, consideram indicadores de reclamações de clientes e a existência de incidentes relacionados aos projetos em questão.

Todos os entrevistados enfatizaram a importância do apoio da gestão para que os projetos alcancem os benefícios previstos, além do controle periódico do cronograma, custo e escopo, também conhecidos como triângulo de ferro. Destaca-se que a literatura considera apenas o controle do cronograma como fator crítico, porém percebe-se que na empresa avaliada, essa que possui um alto nível de maturidade no gerenciamento de riscos, o controle do custo e escopo é de igual importância.

Com relação à adequada conclusão dos projetos, os entrevistados enfatizaram que o fato da área de tecnologia ser focada em projetos, facilita a execução e conclusão satisfatória destes, visto que os processos de gerenciamento de projetos, sejam relacionados às abordagens ágeis ou tradicionais, estão totalmente definidos, disponíveis e disseminados em todos os níveis da organização.

Por fim, foi solicitado aos entrevistados uma priorização em escala de importância, considerando os fatores críticos identificados na Tabela 4, assim como a identificação de algum fator crítico de sucesso que não estivesse descrito na tabela mencionada. Com isso, verificou-se que o comprometimento de gestão, cronograma do projeto e os processos para definição dos projetos, foram considerados os itens mais relevantes, conforme descrito na Tabela 6. Por outro lado, o envolvimento do cliente e a estratégia de entrega, dois dos principais benefícios apontados pela literatura para a abordagem ágil, foram considerados os menos importantes, dentre os fatores críticos avaliados. Destaca-se ainda que, segundo os entrevistados, apenas fatores relacionados ao controle de custo e escopo deveriam ser adicionados à lista identificada na literatura e apresentada na Tabela 4.

\section{Tabela 6}

Ordem de importância dos fatores críticos de sucesso (FCS)

\begin{tabular}{ll}
\hline Ordem & Fator crítico de sucesso \\
\hline 1. & Comprometimento de gestão \\
2. & Cronograma do projeto \\
3. & Processo de definição de projetos \\
4. & Capacidade da equipe \\
5. & Processo de gerenciamento de projetos \\
6. & Técnicas ágeis \\
7. & Ambiente organizacional \\
8. & Ambiente da equipe \\
9. & Natureza do projeto
\end{tabular}




\begin{tabular}{ll}
\hline Ordem & Fator crítico de sucesso \\
\hline 10. & Tipo do projeto \\
11. & Estratégia de entrega \\
12. & Envolvimento do cliente \\
\hline
\end{tabular}

Fonte: Elaborado pelos autores.

Assim, observa-se que, possivelmente, a transição das abordagens tradicionais de gerenciamento de projetos para as abordagens ágeis, em grandes instituições financeiras, requer um período maior de adaptação, visto que a percepção dos envolvidos não condiz totalmente com os benefícios apontados na literatura para as abordagens ágeis. Além disso, constatou-se divergências entre as perspectivas dos colaboradores que exerceram os papéis de Team Lead e Tech Lead, visto que o primeiro mantém o foco nos fatores relacionados aos processos de gerenciamento de projetos, enquanto o segundo direciona seus esforços aos itens relacionados às equipes e processos técnicos. Com isso, a priorização dos fatores críticos apresentados na Tabela 6 , foi realizada com base na quantidade de respostas para cada item, ou seja, a ordem mais citada pelos entrevistados.

Por fim, conclui-se que, apesar de existir algumas divergências, os fatores críticos de sucesso identificados na literatura são, majoritariamente, relevantes e necessários para que grandes instituições financeiras, tal como a empresa avaliada, implantem de maneira satisfatória os projetos de tecnologia gerenciados por abordagens ágeis.

\section{CONCLUSÃO}

Este relato técnico buscou analisar os fatores críticos de sucesso (FCS) de projetos de tecnologia, gerenciados por abordagens ágeis em uma das maiores instituições financeiras da américa latina, com o intuito de verificar se os fatores identificados na literatura, seriam suficientes para que um projeto com essas características fosse implementado com sucesso.

A instituição financeira avaliada possui diversos projetos em seu portfólio, esses que são gerenciados por abordagens tradicionais ou ágeis, dependendo de suas características. Assim, com base nos filtros aplicados, selecionou-se de forma aleatória cinco projetos concluídos e classificados como casos de sucesso.

Desta maneira, pode-se afirmar que o objetivo deste relato foi atingido. Ao observar que, os fatores críticos identificados na literatura são relevantes para o sucesso de um projeto de tecnologia gerenciado por abordagens ágeis. Porém, identificou-se divergências entre os fatores mais importantes identificados na literatura e os fatores classificados como mais relevantes pelos colaboradores entrevistados neste estudo. 
A principal divergência refere-se à importância de um controle apurado das variáveis relacionadas ao custo e escopo, estas que não estão descritas na literatura como um fator crítico de sucesso para projetos gerenciados por abordagens ágeis. Ressalta-se que as variáveis cronograma, custo e escopo, formam as variáveis de eficiência dos projetos, também conhecidas como triângulo de ferro.

Além disso, os fatores relacionados ao envolvimento do cliente e a estratégia de entrega, dois dos principais benefícios apontados na literatura para a abordagem ágil, foram considerados os menos importantes, dentre os fatores críticos avaliados. Por sua vez, o comprometimento da gestão, foi considerado o fator mais relevante para o sucesso de um projeto com as características citadas anteriormente.

As conclusões apresentadas por esta pesquisa devem considerar diversas limitações inerentes às características da empresa avaliada, ao método de pesquisa, aos perfis profissionais dos entrevistados, aos projetos selecionados para avaliação, dentre outras.

Com base nos resultados deste trabalho, identifica-se a oportunidade de novos estudos sobre os fatores de sucesso de projetos gerenciados por abordagens ágeis em instituições financeiras, considerando outras instituições, diferentes tipos de projetos ou mesmo profissionais com perfis distintos, aumentando assim a abrangência de investigação. Além disso, sugere-se estudos futuros sobre os critérios que resultaram na não priorização dos itens relacionados à estratégia de entrega e envolvimento dos clientes, considerando instituições com níveis distintos de maturidade em gerenciamento de projetos, visto que se verificou um aparente paradoxo nesta pesquisa. Ainda, recomenda-se que sejam realizados estudos em outros segmentos da indústria que utilizam as abordagens ágeis para o gerenciamento de projetos, a fim de averiguar se os resultados se repetem em diferentes contextos.

\section{REFERÊNCIAS}

Anderson, D. J. (2003). Agile Management for Software Engineering: Applying the Theory of Constraints for Business Results. Prentice Hall.

Biancolino, C. A., Kniess, C. T., Maccari, E. A., \& Rabechini Jr., R. (2012). Protocolo para Elaboração de Relatos de Produção Técnica. Revista de Gestão e Projetos - GeP, 3(2), 294-307. https://doi.org/10.5585/10.5585

Boehm, B. (1984). Verifying and Validating Software Requirements and Design Specifications. IEEE Softw., 1(1), 75-88. https://doi.org/10.1109/MS.1984.233702

Boehm, B. (1988). A spiral model of software development and enhancement. Computer, 21(5), 61-72. https://doi.org/10.1109/2.59 
Boehm, B. (2000). Requirements that handle IKIWISI, COTS, and rapid change. Computer, 33(7), 99-102. https://doi.org/10.1109/2.869384

Boehm, B. (2002). Get ready for agile methods, with care. Computer, 35(1), 64-69. https://doi.org/10.1109/2.976920

Boehm, B., \& Turner, R. (2003). Using risk to balance agile and plan-driven methods. Computer, 36(6), 57-66. https://doi.org/10.1109/MC.2003.1204376

Chow, T., \& Cao, D.-B. (2008). A survey study of critical success factors in agile software projects. Journal of Systems and Software, 81(6), 961-971. https://doi.org/10.1016/j.jss.2007.08.020

Dybå, T., \& Dingsøyr, T. (2008). Empirical studies of agile software development: A systematic review. Information and Software Technology, 50(9), 833-859. https://doi.org/10.1016/j.infsof.2008.01.006

Gil, A. C. (2010). Como elaborar projetos de pesquisa. Atlas.

Heninger, K. L. (1980). Specifying Software Requirements for Complex Systems: New Techniques and Their Application. IEEE Transactions on Software Engineering, SE-6(1), 2-13. https://doi.org/10.1109/TSE.1980.230208

Highsmith, J., \& Cockburn, A. (2001). Agile software development: The business of innovation. Computer, 34(9), 120-127. https://doi.org/10.1109/2.947100

Kloppenborg, T., Manolis, C., \& Tesch, D. (2009). Successful project sponsor behaviors during project initiation: An empirical investigation. Journal of Managerial Issues, 21, $140-159$.

Martins, G. de A., \& Theóphilo, C. R. (2009). Metodologia da investigação científica para ciências sociais aplicadas. Atlas.

Patah, L. A., \& Carvalho, M. M. de. (2012). Métodos de Gestão de Projetos e Sucesso dos Projetos: Um Estudo Quantitativo do Relacionamento entre estes Conceitos. Revista de Gestão e Projetos - GeP, 3(2), 178-206. https://doi.org/10.5585/10.5585

Paternoster, N., Giardino, C., Unterkalmsteiner, M., Gorschek, T., \& Abrahamsson, P. (2014). Software development in startup companies: A systematic mapping study. Information and Software Technology, 56(10), 1200-1218. https://doi.org/10.1016/j.infsof.2014.04.014

Rockart, J. (1979). Chief Executives Define Their Own Data Needs. Harvard business review, 57, 81-93.

Senapathi, M., \& Drury-Grogan, M. L. (2017). Refining a model for sustained usage of agile methodologies. Journal of Systems and Software, 132, 298-316. https://doi.org/10.1016/j.jss.2017.07.010

Serrador, P., \& Pinto, J. (2015). Does Agile work? - A quantitative analysis of agile project success. International Journal of Project Management, 33(5), 1040-1051. https://doi.org/10.1016/j.ijproman.2015.01.006

Serrador, P., \& Turner, J. R. (2014). The Relationship between Project Success and Project Efficiency. Procedia - Social and Behavioral Sciences, 119, 75-84. https://doi.org/10.1016/j.sbspro.2014.03.011

Shenhar, A., Levy, O., \& Dvir, D. (1997). Mapping the Dimensions of Project Success. https://www.pmi.org/learning/library/mapping-dimensions-project-success-5378

Terlizzi, M., Bento, D., \& Biancolino, C. (2014). Auditoria de Projetos no Banco Itaú. Revista Inovação, Projetos e Tecnologias, 2(1), 98-114. https://doi.org/10.5585/iptec.v2i1.17

The Standish Group. (2015). Standish Group 2015 Chaos Report. InfoQ. https://www.infoq.com/articles/standish-chaos-2015

Turner, J. R. (2008). The Handbook of Project-based Management: Leading Strategic Change in Organizations. McGraw Hill Professional. 
Wit, A. (1988). Measurement of project success. International Journal of Project Management, 6(3), 164-170. https://doi.org/10.1016/0263-7863(88)90043-9 